\title{
miR-486-5p regulates the migration and invasion of colorectal cancer cells through targeting PIK3R1
}

\author{
YUHAO ZHANG ${ }^{1,2}$, JUN FU ${ }^{2}$, ZHIJIN ZHANG ${ }^{2}$ and HUANLONG QIN ${ }^{1}$ \\ ${ }^{1}$ Department of General Surgery, The Affiliated Shanghai No. 10 People's Hospital, Nanjing Medical University, \\ Shanghai 200072; ${ }^{2}$ Department of General Surgery, Shanghai No. 8 People's Hospital, Shanghai 200235, P.R. China
}

Received July 17, 2017; Accepted January 16, 2018

DOI: $10.3892 / \mathrm{ol} .2018 .8233$

\begin{abstract}
The aim of the present study was to investigate the function of microRNA (miR)-486-5p in colorectal cancer (CRC). Tumor and adjacent normal mucosal tissue samples were collected from patients with CRC. Differences in the expression levels of miR-486-5p between tumor tissues and adjacent normal mucosal tissues were examined using reverse transcription-quantitative polymerase chain reaction. The results demonstrated that miR-486-5p was significantly decreased in tumor tissues compared with the adjacent normal mucosal tissues. Additionally, in vitro experiments were conducted using SW620 CRC cells. The effects of miR-486-5p mimics on cell invasion and cell migration were evaluated using a Transwell assay and a wound-healing assay, respectively. The results demonstrated that treatment with miR-486-5p mimics decreased the migratory and invasive ability of the cells compared with that in the blank and NC control groups, although the underlying molecular mechanisms were not determined. Protein expression levels of phosphatidylinositol 3-kinase regulatory subunit 1 (PIK3R1), matrix metallopeptidases-2 and -9 , and phosphorylated (p)-AKT were examined using western blot analysis. The results demonstrated that the expression levels of these proteins decreased in response to treatment with miR-486-5p mimics in comparison with the blank and NC control groups. Taken together, the findings of the present study indicated that miR-486-5p mimics inhibited the progression of CRC by inhibiting the activation of AKT signaling pathway via targeting PIK3R1. Therefore, miR-486-5p may be a potential target for CRC treatment.
\end{abstract}

Correspondence to: Dr Huanlong Qin, Department of General Surgery, The Affiliated Shanghai No. 10 People's Hospital, Nanjing Medical University, 301 Yanchang Road, Zhabei, Shanghai 200072, P.R. China

E-mail: qinhuanlongtph@163.com

Key words: microRNA-486-5p, colorectal cancer, phosphatidylinositol 3-kinase regulatory subunit 1

\section{Introduction}

Colorectal cancer (CRC) accounts for 1.2 million new cases of cancer and 700,000 mortalities annually. CRC is the fourth leading cause of cancer-associated mortality worldwide (1). CRC remains a major public health problem in the majority of industrialized countries (2). Poor treatment efficiency and a lack of treatment options for metastatic CRC are the main causes of CRC-associated mortality. The 5-year survival rate for patients with localized CRC is $90.3 \%$. However, the rate decreases to 70.4 and $12.5 \%$ with regional and distant metastasis, respectively (3). The increasing incidence of CRC in China may be attributed to increased fat intake and decreased physical activity, characteristics of a western lifestyle (4). Despite advances in medical research, the molecular mechanisms underlying the development of CRC require further investigation.

microRNAs (miRNAs/miRs) are small, non-coding RNAs of 19-25 nucleotides that may negatively regulate gene expression at a transcriptional or post-transcriptional level (5). Additionally, the expression profiles of miRNAs may be more accurate in classifying subtypes of tumors compared with mRNA expression profiles (6). Previous studies demonstrated the importance of miRNAs during the development, classification, diagnosis and prognosis of CRC (7-9). miR-486-5p has been reported to function as a tumor suppressor in various types of cancer, including non-small-cell lung (10) and primary gastric cancer (11).

The aim of present study was to examine the function of miR-486-5p in CRC and investigate novel therapeutic targets for CRC.

\section{Materials and methods}

Patients and specimens. Tumor tissue samples and paired normal mucosal tissues were obtained from 25 patients (16 male and 9 female) who were aged between 35 and 62 years old with a mean age of $46 \pm 7$ years old and underwent surgery between February 2014 and January 2016 at the Affiliated Shanghai No. 10 People's Hospital of Nanjing Medical University (Shanghai, China). Samples were stored at $-80^{\circ} \mathrm{C}$. The diagnosis of CRC was confirmed by three pathologists. The present study was approved by the ethical committee of the Affiliated Shanghai No. 10 People's Hospital of Nanjing 
Medical University. Informed consent was obtained from all patients. Patients who did not receive chemotherapy or radiotherapy prior to surgery were enrolled in the present study. As for histological grade, there were 15 patients who were at a well/moderate grade and 10 patients who were at a poor grade. As for Tumor Node Metastasis stage, there were 11 patients who were at a I/II stage and 14 patients who were at a III/IV stage, which were categorized as previously reported (12).

Cell culture and reagents. NCM640 normal human colon epithelial cells and human CRC SW620 and HT-29 cells were obtained from Boster Biological Technology (Pleasanton, CA, USA). Cells were maintained in RPMI-1640 supplemented with $10 \%$ fetal bovine serum (FBS; both Thermo Fisher Scientific, Inc., Waltham, MA, USA) at $37^{\circ} \mathrm{C}$ in a humidified atmosphere containing $5 \% \mathrm{CO}_{2}$.

Lentiviral vectors expressing firefly luciferase were purchased from Hanbio Biotechnology Co., Ltd. (Shanghai, China). miR-486-5p mimics (cat. no. C08002) and miRNA negative controls (NC) (cat. no. B04002) were synthesized by Shanghai GenePharma Co., Ltd. (Shanghai, China) and dissolved in diethylpyrocarbonate water.

Transfection. SW620 cells were transfected with $50 \mathrm{nmol} / \mathrm{l}$ miR-486-5p mimics or NC using Lipofectamine ${ }^{\circledR} 2000$ (Thermo Fisher Scientific, Inc.) according to the manufacturer's protocol. Cells were seeded in a 6-well plate and incubated until they reached $60-70 \%$ confluence. At $24 \mathrm{~h}$, cells were harvested to evaluate the transfection efficiency by reverse transcription-quantitative polymerase chain reaction (RT-qPCR). Transfected cells were employed in the subsequent experiments.

$R T-q P C R$. Total RNA from the cells and tissues was isolated using an RNAiso Plus kit (Takara Biotechnology Co., Ltd., Dalian, China) according to the manufacturer's protocol. RNA quality was evaluated using NanoDrop 1000 spectrophotometer (Thermo Fisher Scientific, Inc., Wilmington, DE, USA). RNA was reversed-transcribed using the PrimeScript ${ }^{\mathrm{TM}}$ II first-strand cDNA Synthesis kit (Takara Biotechnology Co., Ltd.). qPCR was performed using a SYBR-Green PCR kit (Takara Biotechnology Co., Ltd.) and the StepOne Plus Real-Time PCR system (Thermo Fisher Scientific, Inc.). Conditions for amplifications were as following: $95^{\circ} \mathrm{C}$ for $2 \mathrm{~min}$, followed by $95^{\circ} \mathrm{C}$ for $15 \mathrm{sec}, 58^{\circ} \mathrm{C}$ for $30 \mathrm{sec}$ and $72^{\circ} \mathrm{C}$ for $30 \mathrm{sec}$, for 40 cycles. Primers for miR-486-5p (sense, 5'-GTACTGAGCTGCCCCGAGAAA-3', antisense Uni-miR qPCR Primer) and U6 (sense, 5'CTCGCT TCGGCAGCACA3' and antisense, 5'ACGCTTCACGAA TTTGCGT3') were synthesized by Guangzhou RiboBio Co., Ltd. (Guangzhou, China), and U6 acted as an internal control. The expression of miR-486-5p was analyzed using the $2^{-\Delta \Delta \mathrm{Cq}}$ method (13).

Western blot analysis. Total proteins were isolated from cells using Mammalian Protein Extraction reagent (Thermo Fisher Scientific, Inc.) including 1\% protease inhibitor cocktail (Merck KGaA, Darmstadt, Germany). The protein concentration was determined using the bicinchoninic acid assay method (Thermo Fisher Scientific, Inc.). A total of $10 \mu \mathrm{g}$ protein per lane was separated using SDS-PAGE (12\% agarose) and transferred onto polyvinylidene difluoride membranes (Bio-Rad Laboratories, Inc., Hercules, CA, USA) followed by blocking using $7 \%$ non-fat milk at room temperature for $1 \mathrm{~h}$. Membranes were then incubated with primary antibodies at $4^{\circ} \mathrm{C}$ overnight, including anti-phosphatidylinositol 3-kinase regulatory subunit 1 (PIK3R1; cat. no. ab191606; 1:1,000; Abcam, Cambridge, MA, USA), anti-matrix metallopeptidase (MMP) 2 (cat. no. ab37150; 1:1,000; Abcam), anti-MMP9 (cat. no. ab76003; 1:1,000; Abcam), anti-phosphorylated (p)-AKT (cat. no. ab38449; 1:1,000; Abcam) and anti-GAPDH (cat. no. ab8245; 1:1,000; Abcam) (loading control), followed by incubation with the goat anti-rabbit horseradish peroxidase-conjugated secondary antibodies (cat. no. ab97080; 1:5,000; Abcam) at room temperature for $2 \mathrm{~h}$. The proteins were detected using enhanced chemiluminescence (Thermo Fisher Scientific, Inc.).

Transwell invasion assay. Cells were re-suspended in $200 \mu \mathrm{l}$ serum-free RPMI-1640 and placed in the upper chamber of 24-well Transwell plates ( $8 \mu \mathrm{m}$-pore; Corning Incorporated, Corning, NY, USA). RPMI-1640 containing 10\% FBS as a chemoattractant was added to the lower chamber. The Transwell membranes were pre-coated with Matrigel. At $48 \mathrm{~h}$, non-invasive cells in the upper chamber were removed by cotton swabs, invasive cells in the bottom chamber were fixed using $70 \%$ ethanol at room temperature for $15 \mathrm{~min}$ and stained using $0.1 \%$ crystal violet (Beijing Solarbio Science \& Technology Co., Ltd., Beijing, China) at room temperature for $20 \mathrm{~min}$. Images were captured under an optical microscope and at least 5 random fields were counted per insert.

Wound-healing assay. A wound-healing assay was performed to examine the migratory ability of cells. Transfected cells were cultured at $37^{\circ} \mathrm{C}$ in a humidified atmosphere containing $5 \% \mathrm{CO}_{2}$ for $24 \mathrm{~h}$. The cell monolayer was scratched using a 20- $\mu \mathrm{l}$ pipette tip and the cells were cultured for further $24 \mathrm{~h}$. The extent of migration was then examined using phase contrast microscopy.

Dual-luciferase reporter assay. TargetScan (http://www. targetscan.org/) was used to identify the potential targets of miR-486-5p. SW620 cells were seeded into 24-well plates and transfected with wild-type (WT) or mutant luciferase reporter plasmids, and miR-486-5p mimics or NC mimics, using Lipofectamine 2000 according to the manufacturer's protocol. At $24 \mathrm{~h}$, relative firefly and Renilla luciferase activity was determined using a Dual-Luciferase Reporter Assay kit (Promega Corporation, Madison, WI, USA) according to the manufacturer's protocol. Firefly luciferase activity was normalized to Renilla luciferase.

Statistical analysis. Data were analyzed using GraphPad Prism (version 6.0; GraphPad Software, Inc., La Jolla, CA, USA). Data were expressed as the mean \pm standard error of the mean. One-way analysis of variance followed by Newman-Keuls post-hoc analysis was used to examine differences among multiple groups. $\mathrm{P}<0.05$ was considered to indicate a statistically significant difference. 
A

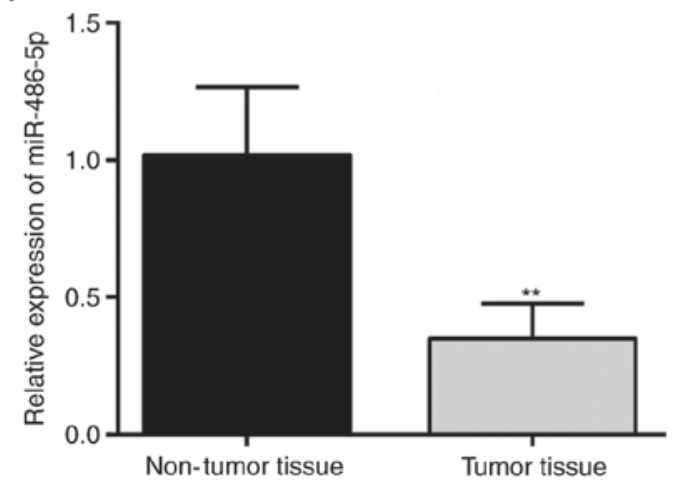

B

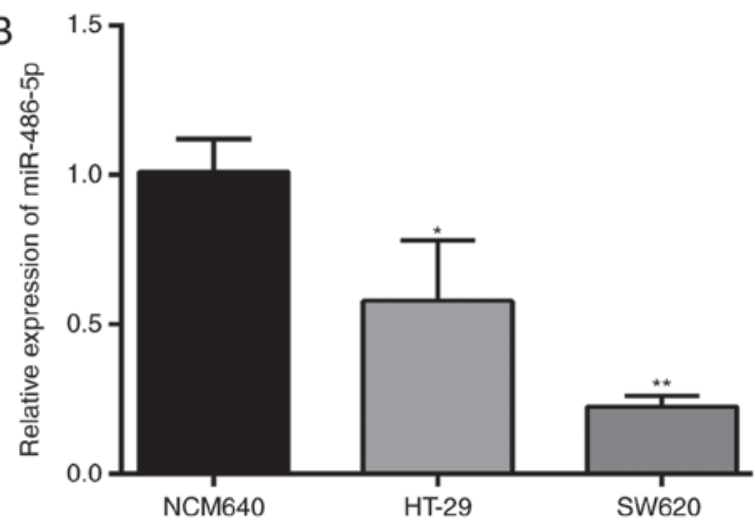

Figure 1. Downregulation of miR-486-5p in CRC tissues and cell lines. (A) miR-486-5p expression was significantly decreased in CRC tissue compared with the adjacent non-tumor tissues. ${ }^{* *} \mathrm{P}<0.01$ vs. non-tumor tissue. (B) miR-486-5p expression was significantly decreased in SW620 and HT-29 CRC cells relative to NCM640 normal human colon epithelial cells. SW620 cells exhibited the lowest miR-486-5p expression levels. ${ }^{*} \mathrm{P}<0.05,{ }^{* *} \mathrm{P}<0.01$ vs. NCM640 cells. miR, microRNA; CRC, colorectal cancer.

\section{Results}

miR-486-5p is downregulated in CRC tissues and cell lines. miR-486-5p has been reported to function as a tumor suppressor in various types of cancer. For example, miR-486-5p was reported to be inversely associated with advanced stage and lymph node metastasis of non-small-cell lung cancer (10), and the levels of miR-486-5p were reported to be decreased in primary gastric cancer and gastric cancer cell lines (11). Therefore, the expression level of miR-486-5p in CRC tissues and cell lines was assessed in the present study using RT-qPCR. The results demonstrated that the expression levels of miR-486-5p were significantly downregulated in CRC tissues compared with that in the adjacent non-tumor tissue (Fig. 1A). Additionally, the expression levels of miR-486-5p were examined in SW620 and HT-29 CRC cell lines, and NCM640 normal human colon epithelial cells. The results demonstrated that the miR-486-5p expression level was significantly decreased in the SW620 and HT-29 cell lines compared with NCM640 (Fig. 1B). SW620 cells exhibited the lowest expression levels of miR-486-5p among the cell lines (Fig. 1B), and thus SW620 cells were employed for the subsequent experiments. These results suggest that miR-486-5p may be involved in the development and progression of CRC.

Evaluation of the transfection efficiency of miR-486-5p mimics into SW620 cells. The effects of miR-486-5p overexpression were evaluated using miR-486-5p mimics in SW620 cells. Transfected SW620 cells were divided into three groups: Blank control, NC-transfected and miR-486-5p mimics group.

The expression levels of miR-486-5p expression in SW620 cells were determined using RT-qPCR. The results demonstrated that the expression levels of miR-486-5p were significantly increased in the miR-486-5p mimics group compared with that in the control groups (Fig. 2), confirming the successful transfection of miR-486-5p mimics into SW620 cells.

Identification of a miR-486-5p target in the PIK3R1 3'UTR. Previous studies have demonstrated that human PIK3R1,

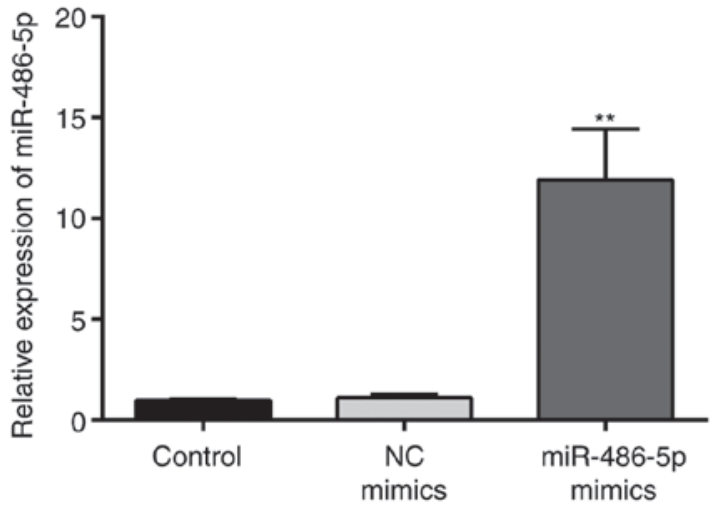

Figure 2. Transfection efficiency of miR-486-5p mimics into SW620 cells As determined by reverse transcription-quantitative polymerase chain reaction, the expression levels of miR-486-5p were significantly increased in the miR-486-5p mimics-transfected group compared with the control groups. ${ }^{* *} \mathrm{P}<0.01$ vs. $\mathrm{NC}$ and blank control groups. miR, microRNA; NC, negative control.

a member of the phosphoinositide 3-kinase (PI3K)-AKT pathway, may be involved in the development of cancer (14-18), including in CRC (19). TargetScan was employed for the identification of potential targets for miR-486-5p. Fig. 3 presents the predicted binding sites of miR-486-5p in the 3'UTR of PIK3R1 (position, 956-963).

A dual-luciferase reporter assay was employed to determine whether miR-486-5p directly targeted the PIK3R1 3'UTR. Reporter constructs containing either the WT or mutant PIK3R1 3'UTR were co-transfected into SW620 cells together with the NC or miR-486-5p mimics. The results demonstrated that transduction of miR-486-5p mimics significantly decreased the luciferase activity of the WT PIK3R1 3'UTR in SW620 cells (Fig. 3B), whereas the activity of the mutant 3'UTR was unaffected. These results confirm that miR-486-5p may directly target PIK3R1.

miR-486-5p mimics inhibit the migration of SW620 cells. The effect of miR-486-5p on the migratory ability of SW620 cells was evaluated using a wound-healing assay. The results demonstrated that there were no differences in the migration 
A

Position 956-963 of PIK3R1 3' UTR 5' ...UUUUUAAAAGUAAAUGUACAGGA...

hsa-miR-486-5p

3' GAGCCCCGUCGAGUCAUGUCCU

Mutant position of PIK3R1 3' UTR $\quad 5^{\prime}$...UUUUUAAAAGUAAAUAGCACAAA...

B

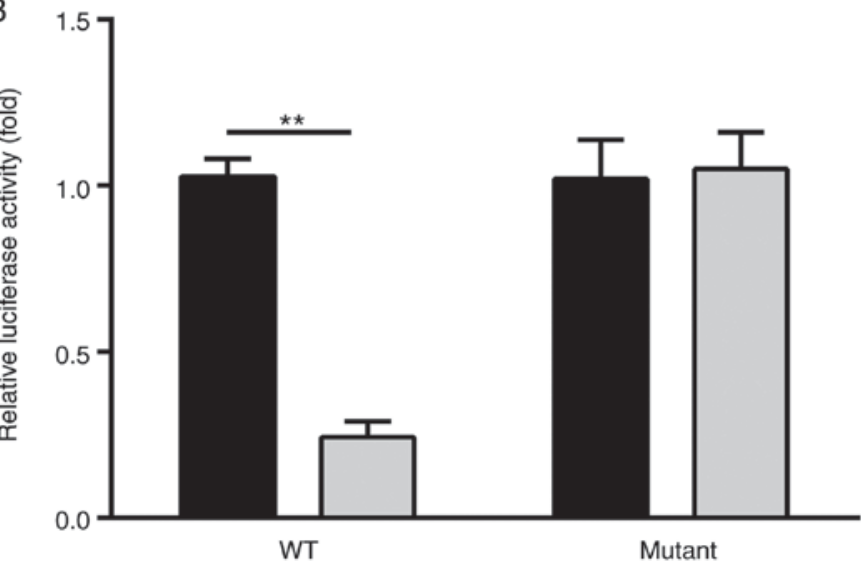

Figure 3. Identification of a miR-486-5p target in the PIK3R1 3'UTR. (A) Schematic representation of the predicted binding site of miR-486-5p within the 3'UTR of PIK3R1 (position, 956-963). (B) Transfection with of miR-486-5p mimics significantly decreased the luciferase activity of the WT PIK3R1 3'UTR in SW620 compared with NC. ${ }^{* *} \mathrm{P}<0.01$ vs. NC. miR, microRNA; PIK3R1, phosphatidylinositol 3-kinase regulatory subunit 1; 3'UTR, 3'-untranslated region; WT, wild-type; NC, negative control.

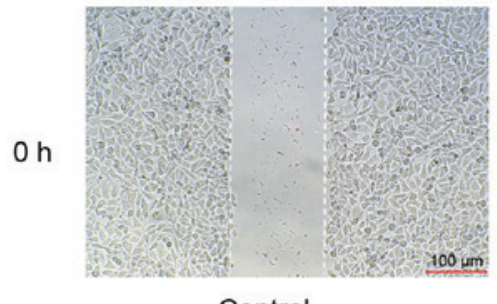

Control

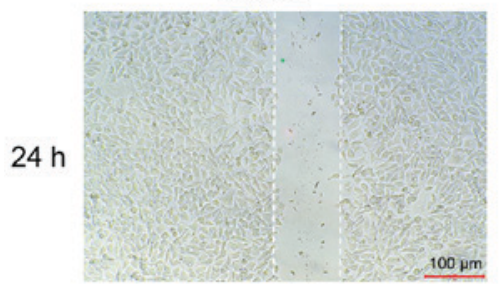

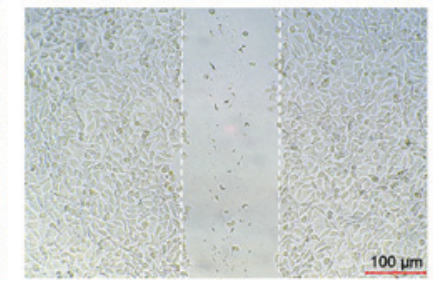

NC mimics

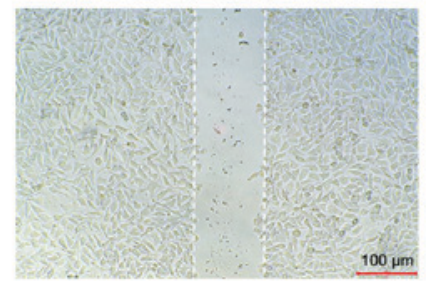

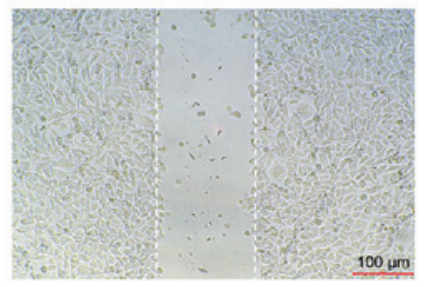

miR-486-5p mimics

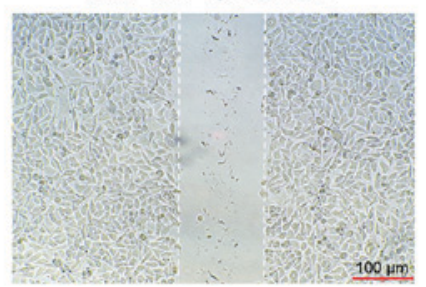

Figure 4. miR-486-5p mimics decrease the migratory ability of SW620 cells. No differences in the migration of cells treated with blank and NC control groups were observed. However, the migration of cells treated with miR-486-5p mimics was decreased compared with that in the control groups. miR, microRNA; NC, negative control.
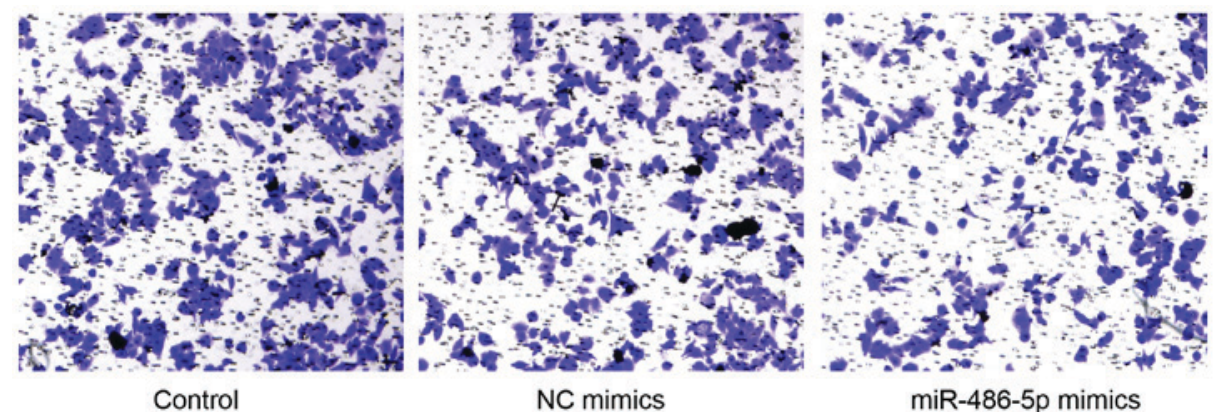

Figure 5. miR-486-5p mimics reduce the invasion of SW620 cells. The invasion of cells treated with miR-486-5p mimics was decreased compared with that in the control groups. miR, microRNA; NC, negative control.

of the blank and $\mathrm{NC}$ control groups. However, the migration of cells treated with miR-486-5p mimics was decreased compared with the control groups (Fig. 4).
miR-486-5p mimics may decrease the invasiveness of SW620 cells. The effect of miR-486-5p on the invasive ability of SW620 cells was examined using a Transwell assay. The 


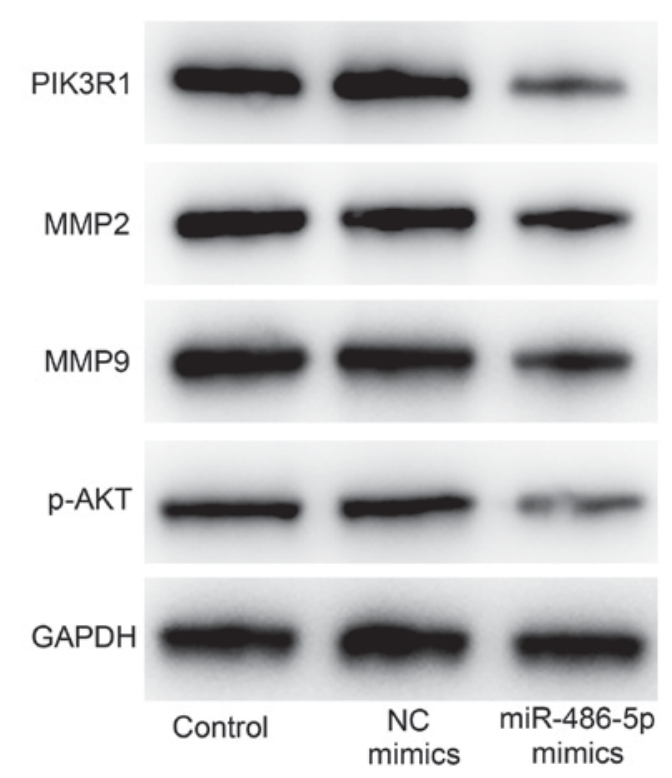

Figure 6. miR-486-5p mimics decrease the expression of $\mathrm{p}-\mathrm{AKT}$, MMP-2/9 and PIK3R1 protein in SW620 cells. The expression levels of p-AKT, MMP-2/9 and PIK3R1 were decreased in cells treated with miR-486-5p mimics compared with the control groups. miR, microRNA; p, phosphorylated; MMP, matrix metalloproteinase; PIK3R1, phosphatidylinositol 3-kinase regulatory subunit 1 ; NC, negative control.

results demonstrated that there were no differences in the invasion of cells treated with blank and NC controls. However, the invasion of cells treated with miR-486-50 mimics was decreased compared with that in the control groups (Fig. 5).

miR-486-5p mimics decrease the expression of MMP-2/9, $p$-AKT and PIK3R1 in SW620 cells. The overexpression of miR-486-5p may inhibit the migration and invasion of CRC cells via PIK3R1 signaling. Therefore, the expression levels of p-AKT and PIK3R1 were evaluated in SW620 cells using western blot analysis. The results demonstrated that the expression of p-AKT and PIK3R in cells treated with miR-486-50 mimics was decreased compared with that in the control groups (Fig. 6).

The MMP family mediates the breakdown of the extracellular matrix under normal physiological conditions, including in cell migration and invasion. MMP is associated with the progression of arthritis and metastasis (20). MMP-2 and -9 may degrade type IV collagen, the most abundant component of the basement membrane, which is an essential step for metastatic progression in the majority of types of cancer (21), and may promote cellular growth and invasion, and tumor progression and metastasis (22). Therefore, the protein levels of MMP-2 and MMP-9 were evaluated in SW620 cells using western blot analysis. The expression of MMP-2 and MMP-9 in cells treated with miR-486-50 mimics was decreased compared with that in the control groups (Fig. 6).

In conclusion, the findings of the present study demonstrated that miR-486-5p mimics may have inhibited the migration and invasion of SW620 CRC cells via targeting PIK3R1 to inhibit the activation of the AKT signaling pathway, and decreasing the expression of MMP-2/9.

\section{Discussion}

CRC is a malignant gastrointestinal tumor of increasing prevalence in China (4). However, the underlying molecular mechanisms remain unclear. miRNAs serve a critical function in various types of cancer (23). A previous study demonstrated that the serum levels of miR-486-5p were downregulated in patients with liver cancer compared with healthy control subjects (24). However, the function of miR-486-5p and its target genes in CRC remain unclear. The results of the present study demonstrated that the levels of miR-486-5p were downregulated in CRC tissues compared with those in adjacent tissues. Additionally, the CRC cell lines exhibited lower expression levels of miR-486-5p compared with NCM640 cells. These results suggest that miR-486-5p may be associated with anticancer activity in CRC.

It was previously demonstrated that the overexpression of miR-486-5p may inhibit cell proliferation, migration and invasion, and decrease tumor growth via targeting PIK3R1 and AKT in hepatocellular carcinoma (25). The results of the present study indicated that the overexpression of miR-486-5p, as simulated using the transfection of miR-486-5p mimics, inhibited cell migration and invasion.

The increased activity of MMP-2 has been associated with the poor prognosis of various types of cancer including CRC, and breast, lung, ovarian and prostate cancer (26). For example, the increased expression of MMP-9 was previously identified in a metastatic mammary cancer cell line (27). Therefore, the present study investigated the expression of MMP-2 and MMP-9 protein using western blot analysis. The results demonstrated that miR-486-5p mimics inhibited the expression of MMP-2 and MMP-9 in SW620 cells.

PIK3R1 was identified as a potential target of miR-486-5p using TargetScan, which was verified with a dual-luciferase reporter assay. In addition, the expression of PIK3R1 and p-AKT protein decreased following transfection of SW620 cells with miR-486-5p mimics compared with that in the control groups.

In conclusion, the decreased expression of miR-486-5p was identified in CRC tissues and cell lines when compared with non-tumor colon tissue samples and cells. Additionally, transfection with miR-486-5p mimics may have decreased the migration and invasion of SW620 cells by targeting PIK3R1. Nevertheless, further investigations on the molecular mechanisms of miR-486-5p in CRC are required. The data of the present study suggest that miR-486-5p may be a potential therapeutic target in CRC.

\section{Acknowledgements}

Not applicable.

\section{Funding}

No funding was received.

\section{Availability of data and materials}

The analyzed data sets generated during the study are available from the corresponding author on reasonable request. 


\section{Authors' contributions}

YZ designed and performed the experiments and analyzed the data, JF performed the experiments and analyzed the data, $\mathrm{ZZ}$ performed the experiments and analyzed the data, HQ designed the experiments and wrote the manuscript. All authors have read and approved the manuscript.

\section{Ethical approval and consent to participate}

The present study was approved by the ethical committee of the Affiliated Shanghai No. 10 People's Hospital of Nanjing Medical University (Shanghai, China). Informed consent was obtained from all patients.

\section{Consent for publication}

Informed consent was obtained from all patients.

\section{Competing interests}

The authors declare that they have no competing interests.

\section{References}

1. Torre LA, Bray F, Siegel RL, Ferlay J, Lortet-Tieulent J and Jemal A: Global cancer statistics, 2012. CA Cancer J Clin 65: 87-108, 2015

2. Pourhoseingholi MA: Increased burden of colorectal cancer in Asia. World J Gastrointest Oncol 4: 68-70, 2012.

3. Siegel R, Desantis C and Jemal A: Colorectal cancer statistics, 2014. CA Cancer J Clin 64: 104-117, 2014.

4. Byeon JS, Yang SK, Kim TI, Kim WH, Lau JY, Leung WK, Fujita R, Makharia GK, Abdullah M, Hilmi I, et al: Colorectal neoplasm in asymptomatic Asians: A prospective multinational multicenter colonoscopy survey. Gastrointest Endosc 65: 1015-1022, 2007.

5. Bartel DP: MicroRNAs: Genomics, biogenesis, mechanism, and function. Cell 116: 281-297, 2004.

6. Esquela-Kerscher A and Slack FJ: Oncomirs-microRNAs with a role in cancer. Nat Rev Cancer 6: 259-269, 2006.

7. Michael MZ, O'Connor SM, van Holst Pellekaan NG, Young GP and James RJ: Reduced accumulation of specific microRNÂs in colorectal neoplasia. Mol Cancer Res 1: 882-891, 2003.

8. Volinia S, Calin GA, Liu CG, Ambs S, Cimmino A, Petrocca F, Visone R, Iorio M, Roldo C, Ferracin M, et al: A microRNA expression signature of human solid tumors defines cancer gene targets. Proc Natl Acad Sci USA 103: 2257-2261, 2006.

9. Earle JS, Luthra R, Romans A, Abraham R, Ensor J, Yao H and Hamilton SR: Association of microRNA expression with microsatellite instability status in colorectal adenocarcinoma. J Mol Diagn 12: 433-440, 2010.

10. Wang J, Tian X, Han R, Zhang X, Wang X, Shen H, Xue L, Liu Y, Yan X, Shen J, et al: Downregulation of miR-486-5p contributes to tumor progression and metastasis by targeting protumorigenic ARHGAP5 in lung cancer. Oncogene 33: 1181-1189, 2014.

11. Oh HK, Tan AL, Das K, Ooi CH, Deng NT, Tan IB, Beillard E, Lee J, Ramnarayanan K, Rha SY, et al: Genomic loss of $m i R-486$ regulates tumor progression and the OLFM4 antiapoptotic factor in gastric cancer. Clin Cancer Res 17: 2657-2667, 2011.
12. Hari DM, Leung AM, Lee JH, Sim MS, Vuong B, Chiu CG and Bilchik AJ: AJCC Cancer Staging Manual 7th edition criteria for colon cancer: Do the complex modifications improve prognostic assessment? J Am Coll Surg 217: 181-90, 2013.

13. Livak KJ and Schmittgen TD: Analysis of relative gene expression data using real-time quantitative PCR and the $2^{-\Delta \Delta C \mathrm{~T}}$ method. Methods 25: 402-408, 2001.

14. Gedaly R, Angulo P, Hundley J, Daily MF, Chen C, Koch A and Evers BM: PI-103 and sorafenib inhibit hepatocellular carcinoma cell proliferation by blocking Ras/Raf/MAPK and PI3K-AKT-mTOR pathways. Anticancer Res 30: 4951-4958, 2010.

15. Chen M, Gu J, Delclos GL, Killary AM, Fan Z, Hildebrandt MA, Chamberlain RM, Grossman HB, Dinney CP and Wu X: Genetic variations of the PI3K-AKT-mTOR pathway and clinical outcome in muscle invasive and metastatic bladder cancer patients. Carcinogenesis 31: 1387-1391, 2010.

16. Zhao L and Vogt PK: Class I PI3K in oncogenic cellular transformation. Oncogene 27: 5486-5496, 2008.

17. Urick ME, Rudd ML, Godwin AK, Sgroi D, Merino M and Bell DW: PIK3R1 (p85 $\alpha$ ) is somatically mutated at high frequency in primary endometrial cancer. Cancer Res 71: 4061-4067, 2011.

18. Huang Y, Gao S, Wu S, Song P, Sun X, Hu X, Zhang S, Yu Y, Zhu J, Li C, et al: Multilayered molecular profiling supported themonoclonal origin of metastatic renal cell carcinoma. Int J Cancer 135: 78-87, 2014.

19. Philp AJ, Campbell IG, Leet C, Vincan E, Rockman SP, Whitehead RH, Thomas RJ and Phillips WA: The phosphatidylinositol 3'-kinase p85alpha gene is an oncogene in human ovarian and colon tumors. Cancer Res 61: 7426-7429, 2001.

20. Vandooren J, Van den Steen PE and Opdenakker G: Biochemistry and molecular biology of gelatinase B or matrix metalloproteinase-9 (MMP-9): The next decade. Crit Rev Biochem Mol Biol 48: 222-272, 2013.

21. Mook OR, Frederiks WM and Van Noorden CJ: The role of gelatinases in colorectal cancer progression and metastasis. Biochim Biophys Acta 1705: 69-89, 2004.

22. Groblewska M, Siewko M, Mroczko B and Szmitkowski M: The role of matrix metalloproteinases (MMPs) and their inhibitors (TIMPs) in the development of esophageal cancer. Folia Histochem Cytobiol 50: 12-19, 2012.

23. Tutar L, Tutar E and Tutar Y: MicroRNAs and cancer; an overview. Curr Pharm Biotechnol 15: 430-437, 2014.

24. Zheng QF, Zhang JY, Wu JS, Zhang Y, Liu M, Bai L, Zhang JY, Zhao J, Chen Y, Duan ZP and Zheng SJ: Upregulation of miRNA-130a represents good prognosis in patients With HBV-related acute-on-chronic liver failure: A prospective study. Medicine 95: e2639, 2016.

25. Huang XP, Hou J, Shen XY, Huang CY, Zhang XH, Xie YA and Luo XL: MicroRNA-486-5p, which is downregulated in hepatocellular carcinoma, suppresses tumor growth by targeting PIK3R1. FEBS J 282: 579-594, 2015.

26. Björklund $M$ and Koivunen E: Gelatinase-mediated migration and invasion of cancer cells. Biochim Biophys Acta 1755: 37-69, 2005.

27. Morini M, Mottolese M, Ferrari N, Ghiorzo F, Buglioni S, Mortarini R, Noonan DM, Natali PG and Albini A: The alpha 3 beta 1 integrin is associated with mammary carcinoma cell metastasis, invasion, and gelatinase B (mmp-9) activity. Int J Cancer 87: 336-342, 2000. 